\title{
Barnes-type Peters polynomial with umbral calculus viewpoint
}

\author{
Dae San Kim ${ }^{1}$,Taekyun Kim²* ${ }^{2}$ Hyuck In Kwon², Toufik Mansour ${ }^{3}$ and Jongjin Seo ${ }^{4}$
}

"Correspondence: tkkim@kw.ac.kr 2Department of Mathematics, Kwangwoon University, Seoul, 139-701, S. Korea

Full list of author information is available at the end of the article

\begin{abstract}
In this paper, we consider the Barnes-type Peters polynomials. We present several explicit formulas and recurrence relations for these polynomials. Also, we establish a connection between our polynomials and several known families of polynomials.
\end{abstract}

MSC: 05A40; 11B83

Keywords: Barnes-type Peters polynomials; umbral calculus

\section{Introduction}

The aim of this paper is to use umbral calculus to obtain several new and interesting identities of Barnes-type Peters polynomials. Umbral calculus has been used in numerous problems of mathematics (for example, see [1-10]). Umbral techniques have been used in different areas of physics; for example, it was used in group theory and quantum mechanics by Biedenharn et al. [11, 12] (for other examples, see [3, 10, 13-18]).

Let $r \in \mathbb{Z}_{>0}$. Here we will consider the polynomials $S_{n}(x)=S_{n}\left(x \mid \lambda_{1}, \ldots, \lambda_{r} ; \mu_{1}, \ldots, \mu_{r}\right)$ and $\hat{S}_{n}(x)=\hat{S}_{n}\left(x \mid \lambda_{1}, \ldots, \lambda_{r} ; \mu_{1}, \ldots, \mu_{r}\right)$, which are called Barnes-type Peters polynomials of the first kind and of the second kind, respectively, and are given by

$$
\begin{aligned}
& \prod_{j=1}^{r}\left(1+(1+t)^{\lambda_{j}}\right)^{-\mu_{j}}(1+t)^{x}=\sum_{n \geq 0} S_{n}(x) \frac{t^{n}}{n !}, \\
& \prod_{j=1}^{r}\left(\frac{(1+t)^{\lambda_{j}}}{1+(1+t)^{\lambda_{j}}}\right)^{\mu_{j}}(1+t)^{x}=\sum_{n \geq 0} \hat{S}_{n}(x) \frac{t^{n}}{n !},
\end{aligned}
$$

where $\lambda_{1}, \ldots, \lambda_{r}, \mu_{1}, \ldots, \mu_{r} \in \mathbb{C}$ with $\lambda_{1}, \ldots, \lambda_{r} \neq 0$. If $r=1$, then these polynomials are generalizations of Boole polynomials, see [19]. If $\mu_{1}=\cdots=\mu_{r}=1$, then $S_{n}(x \mid \lambda)=$ $S_{n}\left(x \mid \lambda_{1}, \ldots, \lambda_{r}\right)=S_{n}\left(x \mid \lambda_{1}, \ldots, \lambda_{r} ; 1, \ldots, 1\right)$ and $\hat{S}_{n}(x \mid \lambda)=\hat{S}_{n}\left(x \mid \lambda_{1}, \ldots, \lambda_{r}\right)=\hat{S}_{n}\left(x \mid \lambda_{1}, \ldots, \lambda_{r} ;\right.$ $1, \ldots, 1)$ are called Barnes-type Boole polynomials of the first kind and of the second kind. So,

$$
\begin{aligned}
& \prod_{j=1}^{r}\left(1+(1+t)^{\lambda_{j}}\right)^{-1}(1+t)^{x}=\sum_{n \geq 0} S_{n}(x \mid \lambda) \frac{t^{n}}{n !}, \\
& \prod_{j=1}^{r}\left(\frac{(1+t)^{\lambda_{j}}}{1+(1+t)^{\lambda_{j}}}\right)(1+t)^{x}=\sum_{n \geq 0} \hat{S}_{n}(x \mid \lambda) \frac{t^{n}}{n !} .
\end{aligned}
$$

O2014 Kim et al.; licensee Springer. This is an Open Access article distributed under the terms of the Creative Commons Attribution License (http://creativecommons.org/licenses/by/2.0), which permits unrestricted use, distribution, and reproduction in any medium, provided the original work is properly cited. 
We introduce the polynomials $E_{n}(x \mid \lambda ; \mu)=E_{n}\left(x \mid \lambda_{1}, \ldots, \lambda_{r} ; \mu_{1}, \ldots, \mu_{r}\right)$ with the generating function

$$
\prod_{j=1}^{r}\left(\frac{2}{1+e^{\lambda_{j} t}}\right)^{\mu_{j}} e^{x t}=\sum_{n \geq 0} E_{n}(x \mid \lambda ; \boldsymbol{\mu}) \frac{t^{n}}{n !} .
$$

These polynomials may be called generalized Barnes-type Euler polynomials. When $\mu_{1}=$ $\cdots=\mu_{r}=1, E_{n}(x \mid \lambda)=E_{n}\left(x \mid \lambda_{1}, \ldots, \lambda_{r}\right)=E_{n}\left(x \mid \lambda_{1}, \ldots, \lambda_{r} ; 1, \ldots, 1\right)$ are called the Barnes-type Euler polynomials. If further $\lambda_{1}=\cdots=\lambda_{r}=1, E_{n}^{(r)}(x)=E_{n}(x \mid 1, \ldots, 1 ; 1, \ldots, 1)$ are called the Euler polynomials of order $r$. When $x=0, S_{n}=S_{n}(\lambda ; \mu)=S_{n}(0 \mid \lambda ; \boldsymbol{\mu})$ and $\hat{S}_{n}=\hat{S}_{n}(\boldsymbol{\lambda} ; \boldsymbol{\mu})=$ $\hat{S}_{n}(0 \mid \lambda ; \mu)$ are called Barnes-type Peters numbers of the first kind and of the second kind, respectively.

Let $\Pi$ be the algebra of polynomials in a single variable $x$ over $\mathbb{C}$, and let $\Pi^{*}$ be the vector space of all linear functionals on $\Pi$. We denote the action of a linear functional $L$ on a polynomial $p(x)$ by $\langle L \mid p(x)\rangle$, and we define the vector space structure on $\Pi^{*}$ by

$$
\left\langle c L+c^{\prime} L^{\prime} \mid p(x)\right\rangle=c\langle L \mid p(x)\rangle+c^{\prime}\left\langle L^{\prime} \mid p(x)\right\rangle
$$

where $c, c^{\prime} \in \mathbb{C}$ (see [19-22]). We define the algebra of formal power series in a single variable $t$ to be

$$
\mathcal{H}=\left\{f(t)=\sum_{k \geq 0} a_{k} \frac{t^{k}}{k !} \mid a_{k} \in \mathbb{C}\right\}
$$

The formal power series in the variable $t$ defines a linear functional on $\Pi$ by setting

$$
\left\langle f(t) \mid x^{n}\right\rangle=a_{n} \quad \text { for all } n \geq 0 \text { (see [19-22]). }
$$

By (1.3) and (1.4), we have

$$
\left\langle t^{k} \mid x^{n}\right\rangle=n ! \delta_{n, k} \quad \text { for all } n, k \geq 0 \text { (see [19-22]), }
$$

where $\delta_{n, k}$ is the Kronecker symbol.

Let $f_{L}(t)=\sum_{n \geq 0}\left\langle L \mid x^{n}\right\rangle \frac{t^{n}}{n !}$. From (1.5), we have $\left\langle f_{L}(t) \mid x^{n}\right\rangle=\left\langle L \mid x^{n}\right\rangle$. Thus, the map $L \mapsto f_{L}(t)$ is a vector space isomorphism from $\Pi^{*}$ onto $\mathcal{H}$. Therefore, $\mathcal{H}$ is thought of as a set of both formal power series and linear functionals. We call $\mathcal{H}$ umbral algebra. Umbral calculus is the study of umbral algebra.

The order $O(f(t))$ of the non-zero power series $f(t)$ is the smallest integer $k$ for which the coefficient of $t^{k}$ does not vanish (see [19-22]). If $O(f(t))=1$ (respectively, $O(f(t))=0$ ), then $f(t)$ is called a delta (respectively, an invertible) series. Suppose that $O(f(t))=1$ and $O(g(t))=0$, then there exists a unique sequence $s_{n}(x)$ of polynomials such that $\left\langle g(t)(f(t))^{k} \mid s_{n}(x)\right\rangle=n ! \delta_{n, k}$, where $n, k \geq 0$ [19, Theorem 2.3.1]. The sequence $s_{n}(x)$ is called the Sheffer sequence for $(g(t), f(t))$ which is denoted by $s_{n}(x) \sim(g(t), f(t))$ (see [19-22]). For $f(t) \in \mathcal{H}$ and $p(x) \in \Pi$, we have $\left\langle e^{y t} \mid p(x)\right\rangle=p(y),\langle f(t) g(t) \mid p(x)\rangle=\langle g(t) \mid f(t) p(x)\rangle$ and

$$
f(t)=\sum_{n \geq 0}\left\langle f(t) \mid x^{n}\right\rangle \frac{t^{n}}{n !}, \quad p(x)=\sum_{n \geq 0}\left\langle t^{n} \mid p(x)\right\rangle \frac{x^{n}}{n !}
$$


(see [19-22]). From (1.6), we obtain

$$
\left\langle t^{k} \mid p(x)\right\rangle=p^{(k)}(0), \quad\left\langle 1 \mid p^{(k)}(x)\right\rangle=p^{(k)}(0),
$$

where $p^{(k)}(0)$ denotes the $k$ th derivative of $p(x)$ with respect to $x$ at $x=0$. So, by (1.7), we get that $t^{k} p(x)=p^{(k)}(x)=\frac{d^{k}}{d x^{k}} p(x)$ for all $k \geq 0$ (see [19-22]).

Let $s_{n}(x) \sim(g(t), f(t))$. Then we have

$$
\frac{1}{g(\bar{f}(t))} e^{y \bar{f}(t)}=\sum_{n \geq 0} s_{n}(y) \frac{t^{n}}{n !}
$$

for all $y \in \mathbb{C}$, where $\bar{f}(t)$ is the compositional inverse of $f(t)$ (see [19-22]). For $s_{n}(x) \sim$ $(g(t), f(t))$ and $r_{n}(x) \sim(h(t), \ell(t))$, let

$$
s_{n}(x)=\sum_{k=0}^{n} c_{n, k} r_{k}(x)
$$

then we have

$$
c_{n, k}=\frac{1}{k !}\left\langle\frac{h(\bar{f}(t))}{g(\bar{f}(t))}(\ell(\bar{f}(t)))^{k} \mid x^{n}\right\rangle
$$

(see [19-22]).

It is immediate from (1.1)-(1.2), we see that $S_{n}(x)$ and $\hat{S}_{n}(x)$ are the Sheffer sequences for the pairs

$$
\begin{aligned}
& S_{n}(x) \sim\left(\prod_{j=1}^{r}\left(1+e^{\lambda_{j} t}\right)^{\mu_{j}}, e^{t}-1\right), \\
& \hat{S}_{n}(x) \sim\left(\prod_{j=1}^{r}\left(\frac{1+e^{\lambda_{j} t}}{e^{\lambda_{j} t}}\right)^{\mu_{j}}, e^{t}-1\right) .
\end{aligned}
$$

The aim of the present paper is to present several new identities for the Peters polynomials by the use of umbral calculus.

\section{Explicit expressions}

It is well known that

$$
(x)_{n}=\sum_{m=0}^{n} S_{1}(n, m) x^{m} \sim\left(1, e^{t}-1\right)
$$

where $S_{1}(n, m)$ is the Stirling number of the first kind. By (1.11) and (1.12) we have

$$
\prod_{j=1}^{r}\left(1+e^{\lambda_{j} t}\right)^{\mu_{j}} S_{n}(x) \sim\left(1, e^{t}-1\right) \text { and } \prod_{j=1}^{r}\left(\frac{1+e^{\lambda_{j} t}}{e^{\lambda_{j} t}}\right)^{\mu_{j}} \hat{S}_{n}(x) \sim\left(1, e^{t}-1\right) .
$$


So

$$
\begin{aligned}
S_{n}(x) & =\prod_{j=1}^{r}\left(1+e^{\lambda_{j} t}\right)^{-\mu_{j}}(x)_{n}=\sum_{m=0}^{n} S_{1}(n, m) \prod_{j=1}^{r}\left(1+e^{\lambda_{j} t}\right)^{-\mu_{j}} x^{m} \\
& =2^{-\sum_{j=1}^{r} \mu_{j}} \sum_{m=0}^{n} S_{1}(n, m) \prod_{j=1}^{r}\left(\frac{2}{1+e^{\lambda_{j} t}}\right)^{\mu_{j}} x^{m} \\
& =2^{-\sum_{j=1}^{r} \mu_{j}} \sum_{m=0}^{n} S_{1}(n, m) E_{m}(x \mid \lambda ; \boldsymbol{\mu})
\end{aligned}
$$

which implies

$$
\begin{aligned}
\hat{S}_{n}(x) & =\prod_{j=1}^{r}\left(\frac{e^{\lambda_{j} t}}{1+e^{\lambda_{j} t}}\right)^{\mu_{j}}(x)_{n}=e^{\sum_{j=1}^{r} \lambda_{j} \mu_{j} t} \prod_{j=1}^{r}\left(1+e^{\lambda_{j} t}\right)^{-\mu_{j}}(x)_{n} \\
& =2^{-\sum_{j=1}^{r} \mu_{j}} \sum_{m=0}^{n} S_{1}(n, m) e^{\sum_{j=1}^{r} \lambda_{j} \mu_{j} t} \prod_{j=1}^{r}\left(\frac{2}{1+e^{\lambda_{j} t}}\right)^{\mu_{j}} x^{m} \\
& =2^{-\sum_{j=1}^{r} \mu_{j}} \sum_{m=0}^{n} S_{1}(n, m) e^{\sum_{j=1}^{r} \lambda_{j} \mu_{j} t} E_{m}(x \mid \lambda ; \mu) \\
& =2^{-\sum_{j=1}^{r} \mu_{j}} \sum_{m=0}^{n} S_{1}(n, m) E_{m}\left(x+\sum_{j=1}^{r} \lambda_{j} \mu_{j} \mid \lambda ; \mu\right) .
\end{aligned}
$$

Thus, we have the following result.

Theorem 1 For all $n \geq 0$,

$$
\begin{aligned}
& S_{n}(x)=2^{-\sum_{j=1}^{r} \mu_{j}} \sum_{m=0}^{n} S_{1}(n, m) E_{m}(x \mid \lambda ; \boldsymbol{\mu}), \\
& \hat{S}_{n}(x)=2^{-\sum_{j=1}^{r} \mu_{j}} \sum_{m=0}^{n} S_{1}(n, m) E_{m}\left(x+\sum_{j=1}^{r} \lambda_{j} \mu_{j} \mid \lambda ; \boldsymbol{\mu}\right) .
\end{aligned}
$$

By (1.6), (1.8), (1.11) and (1.12), we have

$$
\begin{aligned}
& S_{n}(x)=\sum_{j=0}^{n} \frac{1}{j !}\left\langle\prod_{j=1}^{r}\left(1+(1+t)^{\lambda_{j}}\right)^{-\mu_{j}}(\log (1+t))^{j} \mid x^{n}\right\rangle x^{j}, \\
& \hat{S}_{n}(x)=\sum_{j=0}^{n} \frac{1}{j !}\left\langle\prod_{j=1}^{r}\left(\frac{(1+t)^{\lambda_{j}}}{1+(1+t)^{\lambda_{j}}}\right)^{\mu_{j}}(\log (1+t))^{j} \mid x^{n}\right\rangle x^{j},
\end{aligned}
$$

where

$$
\begin{aligned}
& \left\langle\prod_{j=1}^{r}\left(1+(1+t)^{\lambda_{j}}\right)^{-\mu_{j}}(\log (1+t))^{j} \mid x^{n}\right\rangle \\
& \quad=\left\langle\prod_{j=1}^{r}\left(1+(1+t)^{\lambda_{j}}\right)^{-\mu_{j}} \mid(\log (1+t))^{j} x^{n}\right\rangle
\end{aligned}
$$




$$
\begin{aligned}
& =j ! \sum_{\ell=j}^{n}\left(\begin{array}{l}
n \\
\ell
\end{array}\right) S_{1}(\ell, j)\left\langle\prod_{j=1}^{r}\left(1+(1+t)^{\lambda_{j}}\right)^{-\mu_{j}} \mid x^{n-\ell}\right\rangle \\
& =j ! \sum_{\ell=j}^{n}\left(\begin{array}{l}
n \\
\ell
\end{array}\right) S_{1}(\ell, j) S_{n-\ell}
\end{aligned}
$$

and

$$
\begin{aligned}
& \left\langle\prod_{j=1}^{r}\left(\frac{(1+t)^{\lambda_{j}}}{1+(1+t)^{\lambda_{j}}}\right)^{\mu_{j}}(\log (1+t))^{j} \mid x^{n}\right\rangle \\
& \quad=\left\langle\prod_{j=1}^{r}\left(\frac{(1+t)^{\lambda_{j}}}{1+(1+t)^{\lambda_{j}}}\right)^{\mu_{j}} \mid(\log (1+t))^{j} x^{n}\right\rangle \\
& \quad=j ! \sum_{\ell=j}^{n}\left(\begin{array}{l}
n \\
\ell
\end{array}\right) S_{1}(\ell, j)\left\langle\prod_{j=1}^{r}\left(\frac{(1+t)^{\lambda_{j}}}{1+(1+t)^{\lambda_{j}}}\right)^{\mu_{j}} \mid x^{n-\ell}\right\rangle \\
& \quad=j ! \sum_{\ell=j}^{n}\left(\begin{array}{l}
n \\
\ell
\end{array}\right) S_{1}(\ell, j) \hat{S}_{n-\ell} .
\end{aligned}
$$

Hence, we can state the following formulas.

Theorem 2 For all $n \geq 0$,

$$
S_{n}(x)=\sum_{j=0}^{n}\left(\sum_{\ell=j}^{n}\left(\begin{array}{l}
n \\
\ell
\end{array}\right) S_{1}(\ell, j) S_{n-\ell}\right) x^{j} \quad \text { and } \quad \hat{S}_{n}(x)=\sum_{j=0}^{n}\left(\sum_{\ell=j}^{n}\left(\begin{array}{l}
n \\
\ell
\end{array}\right) S_{1}(\ell, j) \hat{S}_{n-\ell}\right) x^{j} .
$$

Also, by the definitions, (2.1), (1.11) and (1.12), we have

$$
\begin{aligned}
S_{n}(y) & =\left\langle\sum_{i \geq 0} S_{i}(y) \frac{t^{i}}{i !} \mid x^{n}\right\rangle=\left\langle\prod_{j=1}^{r}\left(1+(1+t)^{\lambda_{j}}\right)^{-\mu_{j}}(1+t)^{y} \mid x^{n}\right\rangle \\
& =\left\langle\prod_{j=1}^{r}\left(1+(1+t)^{\lambda_{j}}\right)^{-\mu_{j}} \mid(1+t)^{y} x^{n}\right\rangle \\
& =\sum_{m=0}^{n}(y)_{m}\left(\begin{array}{c}
n \\
m
\end{array}\right)\left\langle\prod_{j=1}^{r}\left(1+(1+t)^{\lambda_{j}}\right)^{-\mu_{j}} \mid x^{n-m}\right\rangle \\
& =\sum_{m=0}^{n}(y)_{m}\left(\begin{array}{c}
n \\
m
\end{array}\right) S_{n-m}
\end{aligned}
$$

and

$$
\begin{aligned}
\hat{S}_{n}(y) & =\left\langle\sum_{i \geq 0} \hat{S}_{i}(y) \frac{t^{i}}{i !} \mid x^{n}\right\rangle=\left\langle\prod_{j=1}^{r}\left(\frac{(1+t)^{\lambda_{j}}}{1+(1+t)^{\lambda_{j}}}\right)^{\mu_{j}}(1+t)^{y} \mid x^{n}\right\rangle \\
& =\left\langle\prod_{j=1}^{r}\left(\frac{(1+t)^{\lambda_{j}}}{1+(1+t)^{\lambda_{j}}}\right)^{\mu_{j}} \mid(1+t)^{y} x^{n}\right\rangle
\end{aligned}
$$




$$
\begin{aligned}
& =\sum_{m=0}^{n}(y)_{m}\left(\begin{array}{c}
n \\
m
\end{array}\right)\left\langle\prod_{j=1}^{r}\left(\frac{(1+t)^{\lambda_{j}}}{1+(1+t)^{\lambda_{j}}}\right)^{\mu_{j}} \mid x^{n-m}\right\rangle \\
& =\sum_{m=0}^{n}(y)_{m}\left(\begin{array}{l}
n \\
m
\end{array}\right) \hat{S}_{n-m},
\end{aligned}
$$

which implies the following formulas.

Theorem 3 For all $n \geq 0$,

$$
S_{n}(x)=\sum_{j=0}^{n} S_{n-j}\left(\begin{array}{l}
n \\
j
\end{array}\right)(x)_{j} \quad \text { and } \quad \hat{S}_{n}(x)=\sum_{j=0}^{n} \hat{S}_{n-j}\left(\begin{array}{l}
n \\
j
\end{array}\right)(x)_{j} .
$$

More generally, by (2.1) and (2.2) with $p_{n}(x)=\prod_{j=1}^{r}\left(1+e^{\lambda_{j} t}\right)^{\mu_{j}} S_{n}(x)=(x)_{n} \sim\left(1, e^{t}-1\right)$, we obtain that $S_{n}(x+y)=\sum_{j=0}^{b} S_{j}(x)(y)_{n-j}\left(\begin{array}{l}n \\ j\end{array}\right)$, and with $p_{n}(x)=\prod_{j=1}^{r}\left(\frac{1+e^{\lambda_{j} t}}{e^{\lambda_{j} t}}\right)^{\mu_{j}} \hat{S}_{n}(x)=(x)_{n} \sim$ $\left(1, e^{t}-1\right)$, we obtain that $\hat{S}_{n}(x+y)=\sum_{j=0}^{b} \hat{S}_{j}(x)(y)_{n-j}\left(\begin{array}{l}n \\ j\end{array}\right)$, which gives the following corollary.

Corollary 1 For all $n \geq 0$,

$$
S_{n}(x+y)=\sum_{j=0}^{b} S_{j}(x)(y)_{n-j}\left(\begin{array}{l}
n \\
j
\end{array}\right) \quad \text { and } \quad \hat{S}_{n}(x+y)=\sum_{j=0}^{b} \hat{S}_{j}(x)(y)_{n-j}\left(\begin{array}{l}
n \\
j
\end{array}\right) \text {. }
$$

\section{Recurrence relations}

Note that if $a_{n}(x) \sim(g(t), f(t))$, then $f(t) a_{n}(x)=n a_{n-1}(x)$, Thus, by (1.11) and (1.12), we have that $S_{n}(x+1)-S_{n}(x)=\left(e^{t}-1\right) S_{n}(x)=n S_{n-1}(x)$ and $\hat{S}_{n}(x+1)-\hat{S}_{n}(x)=\left(e^{t}-1\right) \hat{S}_{n}(x)=n \hat{S}_{n-1}(x)$, which give the following recurrences.

Proposition 1 For all $n \geq 1$,

$$
S_{n}(x+1)-S_{n}(x)=n S_{n-1}(x) \quad \text { and } \quad \hat{S}_{n}(x+1)-\hat{S}_{n}(x)=n \hat{S}_{n-1}(x) .
$$

Note that for $a_{n}(x) \sim(g(t), f(t))$, we have that $a_{n+1}(x)=\left(x-g^{\prime}(t) / g(t)\right) \frac{1}{f^{\prime}(t)} a_{n}(x)$. In the case (1.11), we obtain $S_{n+1}(x)=x S_{n}(x-1)-e^{-t} \frac{g^{\prime}(t)}{g(t)} S_{n}(x)$ with $g(t)=\prod_{i=1}^{r}\left(1+e^{\lambda_{j} t}\right)^{\mu_{j}}$. Since $\frac{g^{\prime}(t)}{g(t)}=\sum_{i=1}^{r} \frac{\lambda_{i} \mu_{i} e^{\lambda_{i} t}}{1+e^{\lambda_{i} t}}$ and by (2.3), we get

$$
\begin{aligned}
\frac{g^{\prime}(t)}{g(t)} S_{n}(x) & =\sum_{i=1}^{r} \frac{\lambda_{i} \mu_{i} e^{\lambda_{i} t}}{1+e^{\lambda_{i} t}} S_{n}(x)=\sum_{i=1}^{r} \frac{\lambda_{i} \mu_{i} e^{\lambda_{i} t}}{2} \frac{2}{1+e^{\lambda_{i} t}} S_{n}(x) \\
& =\sum_{i=1}^{r}\left(\frac{\lambda_{i} \mu_{i} e^{\lambda_{i} t}}{2} \frac{2}{1+e^{\lambda_{i} t}} 2^{-\sum_{j=1}^{r} \mu_{j}} \sum_{m=0}^{n} S_{1}(n, m) \prod_{j=1}^{r}\left(\frac{2}{1+e^{\lambda_{j} t}}\right)^{\mu_{j}} x^{m}\right) \\
& =2^{-\sum_{j=1}^{r} \mu_{j}} \sum_{m=0}^{n} S_{1}(n, m) \sum_{i=1}^{r}\left(\frac{\lambda_{i} \mu_{i} e^{\lambda_{i} t}}{2} \frac{2}{1+e^{\lambda_{i} t}} \prod_{j=1}^{r}\left(\frac{2}{1+e^{\lambda_{j} t}}\right)^{\mu_{j}} x^{m}\right) \\
& =2^{-\sum_{j=1}^{r} \mu_{j}} \sum_{m=0}^{n} S_{1}(n, m) \sum_{i=1}^{r} \frac{\lambda_{i} \mu_{i}}{2} E_{m}\left(x+\lambda_{i} \mid \lambda ; \mu+e_{i}\right),
\end{aligned}
$$


where $e_{i}=(0, \ldots, 0,1,0, \ldots, 0)$ is a vector with 1 in the $i$ th coordinate. Thus,

$$
S_{n+1}(x)=x S_{n-1}(x)-2^{-1-\sum_{i=1}^{r} \mu_{j}} \sum_{m=0}^{n} \sum_{i=1}^{r} S_{1}(n, m) \lambda_{i} \mu_{i} E_{m}\left(x+\lambda_{i}-1 \mid \lambda ; \mu+e_{i}\right) .
$$

On the other hand, by Theorem 2, we have

$$
\begin{aligned}
\frac{g^{\prime}(t)}{g(t)} S_{n}(x) & =\sum_{i=1}^{r} \frac{\lambda_{i} \mu_{i} e^{\lambda_{i} t}}{1+e^{\lambda_{i} t}} S_{n}(x)=\sum_{i=1}^{r} \frac{\lambda_{i} \mu_{i} e^{\lambda_{i} t}}{2} \frac{2}{1+e^{\lambda_{i} t}} S_{n}(x) \\
& =\sum_{i=1}^{r} \frac{\lambda_{i} \mu_{i} e^{\lambda_{i} t}}{2} \frac{2}{1+e^{\lambda_{i} t}} \sum_{j=0}^{n}\left(\sum_{\ell=j}^{n}\left(\begin{array}{l}
n \\
\ell
\end{array}\right) S_{1}(\ell, j) S_{n-\ell}\right) x^{j} \\
& =\sum_{j=0}^{n}\left(\sum_{\ell=j}^{n}\left(\begin{array}{l}
n \\
\ell
\end{array}\right) S_{1}(\ell, j) S_{n-\ell} \sum_{i=1}^{r} \frac{\lambda_{i} \mu_{i} e^{\lambda_{i} t}}{2} \frac{2}{1+e^{\lambda_{i} t}} x^{j}\right) \\
& =\sum_{j=0}^{n}\left(\sum_{\ell=j}^{n}\left(\begin{array}{l}
n \\
\ell
\end{array}\right) S_{1}(\ell, j) S_{n-\ell} \sum_{i=1}^{r} \frac{\lambda_{i} \mu_{i} e^{\lambda_{i} t}}{2} \lambda_{i}^{j} E_{j}\left(x / \lambda_{i}\right)\right) \\
& =\sum_{j=0}^{n}\left(\sum_{\ell=j}^{n}\left(\begin{array}{l}
n \\
\ell
\end{array}\right) S_{1}(\ell, j) S_{n-\ell} \sum_{i=1}^{r} \frac{\lambda_{i}^{j+1} \mu_{i}}{2} E_{j}\left(1+x / \lambda_{i}\right)\right)
\end{aligned}
$$

(note that $E_{n}(x)=\frac{2}{1+e^{t}} x^{n}=(E+x)^{n}=\sum_{j=0}^{n}\left(\begin{array}{c}n \\ j\end{array}\right) E_{j} x^{n-j}$ and $\left.\frac{2}{1+e^{\lambda_{j}}} x^{j}=\lambda_{i}^{j} E_{i}\left(x / \lambda_{i}\right)\right)$, which implies

$$
S_{n+1}(x)=x S_{n}(x-1)-\sum_{j=0}^{n} \sum_{\ell=j}^{n} \sum_{i=1}^{r} \frac{\lambda_{i}^{j+1} \mu_{i}}{2}\left(\begin{array}{l}
n \\
\ell
\end{array}\right) S_{1}(\ell, j) S_{n-\ell} E_{j}\left(1+(x-1) / \lambda_{i}\right) .
$$

Thus, by (3.1), we can state the following result.

Theorem 4 For all $n \geq 0$,

$$
\begin{aligned}
& S_{n+1}(x)=x S_{n}(x-1)-2^{-1-\sum_{i=1}^{r} \mu_{j}} \sum_{m=0}^{n} \sum_{i=1}^{r} S_{1}(n, m) \lambda_{i} \mu_{i} E_{m}\left(x+\lambda_{i}-1 \mid \lambda ; \mu+e_{i}\right), \\
& S_{n+1}(x)=x S_{n}(x-1)-\sum_{j=0}^{n} \sum_{\ell=j}^{n} \sum_{i=1}^{r} \frac{\lambda_{i}^{j+1} \mu_{i}}{2}\left(\begin{array}{l}
n \\
\ell
\end{array}\right) S_{1}(\ell, j) S_{n-\ell} E_{j}\left(1+(x-1) / \lambda_{i}\right) .
\end{aligned}
$$

As a corollary, we get the following identity.

Corollary 2 For all $n \geq 0$,

$$
\begin{aligned}
2^{-1-\sum_{i=1}^{r} \mu_{j}} \sum_{m=0}^{n} \sum_{i=1}^{r} S_{1}(n, m) \lambda_{i} \mu_{i} E_{m}\left(x+\lambda_{i}-1 \mid \lambda ; \mu+e_{i}\right), \\
=\sum_{j=0}^{n} \sum_{\ell=j}^{n} \sum_{i=1}^{r} \frac{\lambda_{i}^{j+1} \mu_{i}}{2}\left(\begin{array}{l}
n \\
\ell
\end{array}\right) S_{1}(\ell, j) S_{n-\ell} E_{j}\left(1+(x-1) / \lambda_{i}\right) .
\end{aligned}
$$


In the case (1.12), we obtain $\hat{S}_{n+1}(x)=x \hat{S}_{n}(x-1)-e^{-t} \frac{g^{\prime}(t)}{g(t)} \hat{S}_{n}(x)$ with $g(t)=\prod_{i=1}^{r}\left(\frac{1+e^{\lambda_{j} t}}{e^{\lambda_{i} t}}\right)^{\mu_{j}}$. Since $\frac{g^{\prime}(t)}{g(t)}=\sum_{i=1}^{r} \frac{\lambda_{i} \mu_{i} e^{\lambda_{i} t}}{1+e^{\lambda_{i} t}}-\sum_{i=1}^{r} \lambda_{i} \mu_{i}$ and by (2.4), we get

$$
\frac{g^{\prime}(t)}{g(t)} S_{n}(x)=\sum_{i=1}^{r} \frac{\lambda_{i} \mu_{i} e^{\lambda_{i} t}}{1+e^{\lambda_{i} t}} \hat{S}_{n}(x)-\lambda \mu \hat{S}_{n}(x),
$$

where $\lambda \mu=\sum_{j=1}^{r} \lambda_{j} \mu_{j}$ and

$$
\begin{aligned}
& \sum_{i=1}^{r} \frac{\lambda_{i} \mu_{i} e^{\lambda_{i} t}}{1+e^{\lambda_{i} t}} \hat{S}_{n}(x) \\
& \quad=\sum_{i=1}^{r} \frac{\lambda_{i} \mu_{i} e^{\lambda_{i} t}}{2} \frac{2}{1+e^{\lambda_{i} t}} \hat{S}_{n}(x) \\
& \quad=\sum_{i=1}^{r}\left(\frac{\lambda_{i} \mu_{i} e^{\lambda_{i} t}}{2} \frac{2}{1+e^{\lambda_{i} t}} 2^{-\sum_{j=1}^{r} \mu_{j}} \sum_{m=0}^{n} S_{1}(n, m) e^{\sum_{j=1}^{r} \lambda_{j} \mu_{j} t} \prod_{j=1}^{r}\left(\frac{2}{1+e^{\lambda_{j} t}}\right)^{\mu_{j}} x^{m}\right) \\
& =2^{-\sum_{j=1}^{r} \mu_{j}} \sum_{m=0}^{n} S_{1}(n, m) \sum_{i=1}^{r}\left(\frac{\lambda_{i} \mu_{i}}{2} e^{\lambda_{i} t+\sum_{j=1}^{r} \lambda_{j} \mu_{j} t} \frac{2}{1+e^{\lambda_{i} t}} \prod_{j=1}^{r}\left(\frac{2}{1+e^{\lambda_{j} t}}\right)^{\mu_{j}} x^{m}\right) \\
& \quad=2^{-1-\sum_{j=1}^{r} \mu_{j}} \sum_{m=0}^{n} S_{1}(n, m) \sum_{i=1}^{r} \lambda_{i} \mu_{i} E_{n}\left(x+\lambda\left(\mu+e_{i}\right) \mid \lambda ; \mu+e_{i}\right) .
\end{aligned}
$$

So

$$
\begin{aligned}
\hat{S}_{n+1}(x)= & (x+\lambda \mu) \hat{S}_{n}(x-1) \\
& -2^{-1-\sum_{j=1}^{r} \mu_{j}} \sum_{m=0}^{n} S_{1}(n, m) \sum_{i=1}^{r} \lambda_{i} \mu_{i} E_{n}\left(x+\lambda\left(\mu+e_{i}\right)-1 \mid \lambda ; \mu+e_{i}\right) .
\end{aligned}
$$

On the other hand, by Theorem 2, we have

$$
\begin{aligned}
\frac{g^{\prime}(t)}{g(t)} \hat{S}_{n}(x) & =\sum_{i=1}^{r} \frac{\lambda_{i} \mu_{i} e^{\lambda_{i} t}}{1+e^{\lambda_{i} t}} \hat{S}_{n}(x)-\lambda \mu \hat{S}_{n}(x)=\sum_{i=1}^{r} \frac{\lambda_{i} \mu_{i} e^{\lambda_{i} t}}{2} \frac{2}{1+e^{\lambda_{i} t}} \hat{S}_{n}(x)-\lambda \mu \hat{S}_{n}(x) \\
& =\sum_{i=1}^{r} \frac{\lambda_{i} \mu_{i} e^{\lambda_{i} t}}{2} \frac{2}{1+e^{\lambda_{i} t}} \sum_{j=0}^{n}\left(\sum_{\ell=j}^{n}\left(\begin{array}{l}
n \\
\ell
\end{array}\right) S_{1}(\ell, j) \hat{S}_{n-\ell}\right) x^{j}-\lambda \mu \hat{S}_{n}(x) \\
& =\sum_{j=0}^{n}\left(\sum_{\ell=j}^{n}\left(\begin{array}{l}
n \\
\ell
\end{array}\right) S_{1}(\ell, j) \hat{S}_{n-\ell} \sum_{i=1}^{r} \frac{\lambda_{i}^{j+1} \mu_{i}}{2} E_{j}\left(1+x / \lambda_{i}\right)\right)-\lambda \mu \hat{S}_{n}(x) .
\end{aligned}
$$

Therefore, by (3.2), we have the following result.

Theorem 5 For all $n \geq 0$,

$$
\begin{aligned}
\hat{S}_{n+1}(x)= & (x+\lambda \boldsymbol{\mu}) \hat{S}_{n}(x-1) \\
& -2^{-1-\sum_{j=1}^{r} \mu_{j}} \sum_{m=0}^{n} S_{1}(n, m) \sum_{i=1}^{r} \lambda_{i} \mu_{i} E_{n}\left(x+\lambda\left(\boldsymbol{\mu}+e_{i}\right)-1 \mid \lambda ; \mu+e_{i}\right),
\end{aligned}
$$




$$
\hat{S}_{n+1}(x)=(x+\lambda \mu) \hat{S}_{n}(x-1)-\sum_{j=0}^{n} \sum_{\ell=j}^{n} \sum_{i=1}^{r} \frac{\lambda_{i}^{j+1} \mu_{i}}{2}\left(\begin{array}{l}
n \\
\ell
\end{array}\right) S_{1}(\ell, j) \hat{S}_{n-\ell} E_{j}\left(1+(x-1) / \lambda_{i}\right) .
$$

As a corollary, we get the following identity.

Corollary 3 For all $n \geq 0$,

$$
\begin{gathered}
2^{-1-\sum_{j=1}^{r} \mu_{j}} \sum_{m=0}^{n} S_{1}(n, m) \sum_{i=1}^{r} \lambda_{i} \mu_{i} E_{n}\left(x+\lambda\left(\mu+e_{i}\right)-1 \mid \lambda ; \mu+e_{i}\right), \\
=\sum_{j=0}^{n} \sum_{\ell=j}^{n} \sum_{i=1}^{r} \frac{\lambda_{i}^{j+1} \mu_{i}}{2}\left(\begin{array}{l}
n \\
\ell
\end{array}\right) S_{1}(\ell, j) \hat{S}_{n-\ell} E_{j}\left(1+(x-1) / \lambda_{i}\right) .
\end{gathered}
$$

Recall that for $a_{n}(x) \sim(g(t), f(t))$, we have $\frac{d}{d x} a_{n}(x)=\sum_{\ell=0}^{n-1}\left(\begin{array}{l}n \\ \ell\end{array}\right)\left\langle\bar{f}(t) \mid x^{n-\ell}\right\rangle a_{\ell}(x)$. Hence, in the case (1.11), namely $a_{n}(x)=S_{n}(x)$, we have

$$
\begin{aligned}
\left\langle\bar{f}(t) \mid x^{n-\ell}\right\rangle & =\left\langle\log (1+t) \mid x^{n-\ell}\right\rangle \\
& =\left\langle\sum_{m \geq 1} \frac{(-1)^{m-1} x^{m}}{m} \mid x^{n-\ell}\right\rangle=(-1)^{n-\ell-1}(n-\ell-1) !,
\end{aligned}
$$

which implies $d / d x S_{n}(x)=n ! \sum_{\ell=0}^{n-1} \frac{(-1)^{n-\ell-1}}{\ell !(n-\ell)} S_{\ell}(x)$. In the same way, we obtain the case $\hat{S}_{n}(x)$, which leads to the following result.

Theorem 6 For all $n \geq 1$,

$$
\frac{d}{d x} S_{n}(x)=n ! \sum_{\ell=0}^{n-1} \frac{(-1)^{n-\ell-1}}{\ell !(n-\ell)} S_{\ell}(x) \quad \text { and } \quad \frac{d}{d x} \hat{S}_{n}(x)=n ! \sum_{\ell=0}^{n-1} \frac{(-1)^{n-\ell-1}}{\ell !(n-\ell)} \hat{S}_{\ell}(x) .
$$

Now we find another recurrence relation by using the derivative operator. For $n \geq 1$, by (1.11) we have

$$
\begin{aligned}
S_{n}(y)= & \left\langle\sum_{i \geq 0} S_{i}(y) \frac{t^{i}}{i !} \mid x^{n}\right\rangle=\left\langle\prod_{j=1}^{r}\left(1+(1+t)^{\lambda_{j}}\right)^{-\mu_{j}}(1+t)^{y} \mid x^{n}\right\rangle \\
= & \left\langle\frac{d}{d t}\left(\prod_{j=1}^{r}\left(1+(1+t)^{\lambda_{j}}\right)^{-\mu_{j}}(1+t)^{y}\right) \mid x^{n-1}\right\rangle \\
= & \left\langle\frac{d}{d t} \prod_{j=1}^{r}\left(1+(1+t)^{\lambda_{j}}\right)^{-\mu_{j}}(1+t)^{y} \mid x^{n-1}\right\rangle \\
& +\left\langle\prod_{j=1}^{r}\left(1+(1+t)^{\lambda_{j}}\right)^{-\mu_{j}} \frac{d}{d t}(1+t)^{y} \mid x^{n-1}\right\rangle \\
= & \left\langle\frac{d}{d t} \prod_{j=1}^{r}\left(1+(1+t)^{\lambda_{j}}\right)^{-\mu_{j}}(1+t)^{y} \mid x^{n-1}\right\rangle+y S_{n-1}(y-1) .
\end{aligned}
$$


Observe that $\frac{d}{d t} \prod_{j=1}^{r}\left(1+(1+t)^{\lambda_{j}}\right)^{-\mu_{j}}=-\prod_{j=1}^{r}\left(1+(1+t)^{\lambda_{j}}\right)^{\mu_{j}} \sum_{i=1}^{r} \lambda_{i} \mu_{i} \frac{(1+t)^{\lambda_{i}-1}}{1+(1+t)^{\lambda_{i}}}$. Thus,

$$
\begin{aligned}
& \left\langle\frac{d}{d t} \prod_{j=1}^{r}\left(1+(1+t)^{\lambda_{j}}\right)^{\mu_{j}}(1+t)^{y} \mid x^{n-1}\right\rangle \\
& \quad=-\sum_{i=1}^{r} \lambda_{i} \mu_{i}\left\langle\left(1+(1+t)^{\lambda_{i}}\right)^{-1} \prod_{j=1}^{r}\left(1+(1+t)^{\lambda_{j}}\right)^{-\mu_{j}}(1+t)^{y+\lambda_{i}-1} \mid x^{n-1}\right\rangle \\
& =-\sum_{i=1}^{r} \lambda_{i} \mu_{i} S_{n-1}\left(y+\lambda_{i}-1 \mid \lambda ; \mu+e_{i}\right) .
\end{aligned}
$$

Hence,

$$
S_{n}(x)=x S_{n-1}(x-1)-\sum_{i=1}^{r} \lambda_{i} \mu_{i} S_{n-1}\left(x+\lambda_{i}-1 \mid \lambda ; \mu+e_{i}\right) .
$$

Also, for $n \geq 1$, by (1.12) we have

$$
\begin{aligned}
\hat{S}_{n}(y)= & \left\langle\sum_{i \geq 0} \hat{S}_{i}(y) \frac{t^{i}}{i !} \mid x^{n}\right\rangle \\
= & \left\langle\prod_{j=1}^{r}\left(\frac{(1+t)^{\lambda_{j}}}{1+(1+t)^{\lambda_{j}}}\right)^{\mu_{j}}(1+t)^{y} \mid x^{n}\right\rangle \\
= & \left\langle\frac{d}{d t}\left[\prod_{j=1}^{r}\left(\frac{(1+t)^{\lambda_{j}}}{1+(1+t)^{\lambda_{j}}}\right)^{\mu_{j}}(1+t)^{y}\right] \mid x^{n-1}\right\rangle \\
= & \left\langle\frac{d}{d t} \prod_{j=1}^{r}\left(\frac{(1+t)^{\lambda_{j}}}{1+(1+t)^{\lambda_{j}}}\right)^{\mu_{j}}(1+t)^{y} \mid x^{n-1}\right\rangle \\
& +\left\langle\prod_{j=1}^{r}\left(\frac{(1+t)^{\lambda_{j}}}{1+(1+t)^{\lambda_{j}}}\right)^{\mu_{j}} \frac{d}{d t}(1+t)^{y} \mid x^{n-1}\right\rangle \\
= & \left\langle\frac{d}{d t} \prod_{j=1}^{r}\left(\frac{(1+t)^{\lambda_{j}}}{1+(1+t)^{\lambda_{j}}}\right)^{\mu_{j}}(1+t)^{y} \mid x^{n-1}\right\rangle+y \hat{S}_{n-1}(y-1) .
\end{aligned}
$$

Observe that $\frac{d}{d t} \prod_{j=1}^{r}\left(\frac{(1+t)^{\lambda_{j}}}{1+(1+t)^{\lambda_{j}}}\right)^{\mu_{j}}=\prod_{j=1}^{r}\left(\frac{(1+t)^{\lambda_{j}}}{1+(1+t)^{\lambda_{j}}}\right)^{\mu_{j}} \sum_{i=1}^{r} \lambda_{i} \mu_{i}(1+t)^{-\lambda_{i}-1} \frac{(1+t)^{\lambda_{i}}}{1+(1+t)^{\lambda_{i}}}$. So

$$
\begin{aligned}
& \left\langle\frac{d}{d t} \prod_{j=1}^{r}\left(\frac{(1+t)^{\lambda_{j}}}{1+(1+t)^{\lambda_{j}}}\right)^{\mu_{j}}(1+t)^{y} \mid x^{n-1}\right\rangle \\
& =\sum_{i=1}^{r} \lambda_{i} \mu_{i}\left\langle\frac{(1+t)^{\lambda_{i}}}{1+(1+t)^{\lambda_{i}}} \prod_{j=1}^{r}\left(\frac{(1+t)^{\lambda_{j}}}{1+(1+t)^{\lambda_{j}}}\right)^{\mu_{j}}(1+t)^{y-\lambda_{i}-1} \mid x^{n-1}\right\rangle \\
& =\sum_{i=1}^{r} \lambda_{i} \mu_{i} \hat{S}_{n-1}\left(y-\lambda_{i}-1 \mid \lambda ; \mu+e_{i}\right) .
\end{aligned}
$$


Thus,

$$
\hat{S}_{n}(x)=x \hat{S}_{n-1}(x-1)+\sum_{i=1}^{r} \lambda_{i} \mu_{i} \hat{S}_{n-1}\left(x-\lambda_{i}-1 \mid \lambda ; \mu+e_{i}\right)
$$

Hence, by (3.3) and (3.4), we obtain the following result.

Theorem 7 For $n \geq 1$,

$$
\begin{aligned}
& S_{n}(x)=x S_{n-1}(x-1)-\sum_{i=1}^{r} \lambda_{i} \mu_{i} S_{n-1}\left(x+\lambda_{i}-1 \mid \lambda ; \mu+e_{i}\right), \\
& \hat{S}_{n}(x)=x \hat{S}_{n-1}(x-1)+\sum_{i=1}^{r} \lambda_{i} \mu_{i} \hat{S}_{n-1}\left(x-\lambda_{i}-1 \mid \lambda ; \mu+e_{i}\right) .
\end{aligned}
$$

Another result that can be obtained is the following.

Theorem 8 For $n-1 \geq m \geq 1$,

$$
\begin{aligned}
\sum_{\ell=0}^{n-m}\left(\begin{array}{l}
n \\
\ell
\end{array}\right) S_{1}(n-\ell, m) S_{\ell}= & \sum_{\ell=0}^{n-m}\left(\begin{array}{c}
n-1 \\
\ell
\end{array}\right) S_{1}(n-1-\ell, m-1) S_{\ell}(-1) \\
& -\sum_{\ell=0}^{n-1-m}\left(\begin{array}{c}
n-1 \\
\ell
\end{array}\right) S_{1}(n-1-\ell, m) \sum_{i=1}^{r} \lambda_{i} \mu_{i} S_{\ell}\left(\lambda_{i}-1 \mid \lambda ; \mu+e_{i}\right), \\
\sum_{\ell=0}^{n-m}\left(\begin{array}{l}
n \\
\ell
\end{array}\right) S_{1}(n-\ell, m) \hat{S}_{\ell}= & \sum_{\ell=0}^{n-m}\left(\begin{array}{c}
n-1 \\
\ell
\end{array}\right) S_{1}(n-1-\ell, m-1) \hat{S}_{\ell}(-1) \\
& +\sum_{\ell=0}^{n-1-m}\left(\begin{array}{c}
n-1 \\
\ell
\end{array}\right) S_{1}(n-1-\ell, m) \sum_{i=1}^{r} \lambda_{i} \mu_{i} \hat{S}_{\ell}\left(-\lambda_{i}-1 \mid \lambda ; \mu+e_{i}\right) .
\end{aligned}
$$

Proof Because of the similarity in the two cases $S_{n}(x)$ and $\hat{S}_{n}(x)$, we only give the proof of the first identity. In order to prove the first identity, we compute the following in two different ways:

$$
A=\left\langle\prod_{j=1}^{r}\left(1+(1+t)^{\lambda_{j}}\right)^{-\mu_{j}}(\log (1+t))^{m} \mid x^{n}\right\rangle .
$$

On the one hand, it is equal to

$$
\begin{aligned}
A & =\left\langle\prod_{j=1}^{r}\left(1+(1+t)^{\lambda_{j}}\right)^{-\mu_{j}} \mid(\log (1+t))^{m} x^{n}\right\rangle \\
& =\left\langle\prod_{j=1}^{r}\left(1+(1+t)^{\lambda_{j}}\right)^{-\mu_{j}} \mid m ! \sum_{\ell \geq m} S_{1}(\ell, m) \frac{t^{\ell}}{\ell !} x^{n}\right\rangle \\
& =m ! \sum_{\ell=m}^{n} S_{1}(\ell, m)\left(\begin{array}{c}
n \\
\ell
\end{array}\right)\left\langle\prod_{j=1}^{r}\left(1+(1+t)^{\lambda_{j}}\right)^{-\mu_{j}} \mid x^{n-\ell}\right\rangle
\end{aligned}
$$




$$
\begin{aligned}
& =m ! \sum_{\ell=m}^{n} S_{1}(\ell, m)\left(\begin{array}{l}
n \\
\ell
\end{array}\right) S_{n-\ell} \\
& =m ! \sum_{\ell=0}^{n-m} S_{1}(n-\ell, m)\left(\begin{array}{l}
n \\
\ell
\end{array}\right) S_{\ell} .
\end{aligned}
$$

On the other hand,

$$
\begin{aligned}
A= & \left\langle\frac{d}{d t}\left[\prod_{j=1}^{r}\left(1+(1+t)^{\lambda_{j}}\right)^{-\mu_{j}}(\log (1+t))^{m}\right] \mid x^{n-1}\right\rangle \\
= & \left\langle\frac{d}{d t} \prod_{j=1}^{r}\left(1+(1+t)^{\lambda_{j}}\right)^{-\mu_{j}}(\log (1+t))^{m} \mid x^{n-1}\right\rangle \\
& +\left\langle\prod_{j=1}^{r}\left(1+(1+t)^{\lambda_{j}}\right)^{-\mu_{j}} \frac{d}{d t}(\log (1+t))^{m} \mid x^{n-1}\right\rangle .
\end{aligned}
$$

Here,

$$
\begin{aligned}
& \left\langle\prod_{j=1}^{r}\left(1+(1+t)^{\lambda_{j}}\right)^{-\mu_{j}} \frac{d}{d t}(\log (1+t))^{m} \mid x^{n-1}\right\rangle \\
& \quad=m\left\langle\prod_{j=1}^{r}\left(1+(1+t)^{\lambda_{j}}\right)^{-\mu_{j}}(1+t)^{-1} \mid(\log (1+t))^{m-1} x^{n-1}\right\rangle \\
& \quad=m ! \sum_{\ell=m-1}^{n-1} S_{1}(\ell, m-1)\left\langle\prod_{j=1}^{r}\left(1+(1+t)^{\lambda_{j}}\right)^{-\mu_{j}}(1+t)^{-1} \mid \frac{t^{\ell}}{\ell !} x^{n-1}\right\rangle \\
& \quad=m ! \sum_{\ell=0}^{n-m}\left(\begin{array}{c}
n-1 \\
\ell
\end{array}\right) S_{1}(n-1-\ell, m-1)\left\langle\prod_{j=1}^{r}\left(1+(1+t)^{\lambda_{j}}\right)^{-\mu_{j}}(1+t)^{-1} \mid x^{\ell}\right\rangle \\
& \quad=m ! \sum_{\ell=0}^{n-m}\left(\begin{array}{c}
n-1 \\
\ell
\end{array}\right) S_{1}(n-1-\ell, m-1) S_{\ell}(-1)
\end{aligned}
$$

and

$$
\begin{aligned}
\left\langle\frac{d}{d t}\right. & \prod_{j=1}^{r}\left(1+(1+t)^{\lambda_{j}}\right)^{-\mu_{j}}(\log (1+t))^{m}\left|x^{n-1}\right\rangle \\
= & -\sum_{i=1}^{r} \lambda_{i} \mu_{i}\left\langle\left(1+(1+t)^{\lambda_{i}}\right)^{-1} \prod_{j=1}^{r}\left(1+(1+t)^{\lambda_{j}}\right)^{-\mu_{j}}(1+t)^{\lambda_{i}-1} \mid(\log (1+t))^{m} x^{n-1}\right\rangle \\
= & -\sum_{i=1}^{r} \lambda_{i} \mu_{i}\left\langle\left(1+(1+t)^{\lambda_{i}}\right)^{-1} \prod_{j=1}^{r}\left(1+(1+t)^{\lambda_{j}}\right)^{-\mu_{j}}(1+t)^{\lambda_{i}-1} \mid m ! \sum_{\ell \geq m} S_{1}(\ell, m) \frac{t^{\ell}}{\ell !} x^{n-1}\right\rangle \\
= & -m ! \sum_{i=1}^{r} \sum_{\ell=m}^{n-1} \lambda_{i} \mu_{i}\left(\begin{array}{c}
n-1 \\
\ell
\end{array}\right) S_{1}(\ell, m) \\
& \times\left\langle\left(1+(1+t)^{\lambda_{i}}\right)^{-1} \prod_{j=1}^{r}\left(1+(1+t)^{\lambda_{j}}\right)^{-\mu_{j}}(1+t)^{\lambda_{i}-1} \mid x^{n-1-\ell}\right\rangle
\end{aligned}
$$




$$
\begin{aligned}
= & -m ! \sum_{i=1}^{r} \sum_{\ell=0}^{n-1-m} \lambda_{i} \mu_{i}\left(\begin{array}{c}
n-1 \\
\ell
\end{array}\right) S_{1}(n-1-\ell, m) \\
& \times\left\langle\left(1+(1+t)^{\lambda_{i}}\right)^{-1} \prod_{j=1}^{r}\left(1+(1+t)^{\lambda_{j}}\right)^{-\mu_{j}}(1+t)^{\lambda_{i}-1} \mid x^{\ell}\right\rangle \\
= & -m ! \sum_{i=1}^{r} \sum_{\ell=0}^{n-1-m} \lambda_{i} \mu_{i}\left(\begin{array}{c}
n-1 \\
\ell
\end{array}\right) S_{1}(n-1-\ell, m) S_{\ell}\left(\lambda_{i}-1 \mid \lambda ; \boldsymbol{\mu}+e_{i}\right) .
\end{aligned}
$$

Altogether, we have, for $n-1 \geq m \geq 1$,

$$
\begin{aligned}
& m ! \sum_{\ell=0}^{n-m}\left(\begin{array}{c}
n \\
\ell
\end{array}\right) S_{1}(n-\ell, m) S_{\ell} \\
& =m ! \sum_{\ell=0}^{n-m}\left(\begin{array}{c}
n-1 \\
\ell
\end{array}\right) S_{1}(n-1-\ell, m-1) S_{\ell}(-1) \\
& \quad-m ! \sum_{i=1}^{r} \sum_{\ell=0}^{n-1-m} \lambda_{i} \mu_{i}\left(\begin{array}{c}
n-1 \\
\ell
\end{array}\right) S_{1}(n-1-\ell, m) S_{\ell}\left(\lambda_{i}-1 \mid \lambda ; \mu+e_{i}\right) .
\end{aligned}
$$

By dividing by $m$ !, we complete the proof.

\section{Identities}

Let $S_{n}(x)=\sum_{m=0}^{n} c_{n, m}(x)_{m}$ and $\hat{S}_{n}(x)=\sum_{m=0}^{n} \hat{c}_{n, m}(x)_{m}$. By (1.9), (1.10) and (1.11), we obtain

$$
\begin{aligned}
c_{n, m} & =\frac{1}{m !}\left\langle\prod_{j=1}^{r}\left(1+(1+t)^{\lambda_{j}}\right)^{-\mu_{j}} \mid t^{m} x^{n}\right\rangle \\
& =\left(\begin{array}{c}
n \\
m
\end{array}\right)\left\langle\prod_{j=1}^{r}\left(1+(1+t)^{\lambda_{j}}\right)^{-\mu_{j}} \mid x^{n-m}\right\rangle \\
& =\left(\begin{array}{c}
n \\
m
\end{array}\right) S_{n-m},
\end{aligned}
$$

and by (1.9), (1.10) and (1.12), we obtain

$$
\begin{aligned}
\hat{c}_{n, m} & =\frac{1}{m !}\left\langle\prod_{j=1}^{r}\left(\frac{(1+t)^{\lambda_{j}}}{1+(1+t)^{\lambda_{j}}}\right)^{\mu_{j}} \mid t^{m} x^{n}\right\rangle \\
& =\left(\begin{array}{c}
n \\
m
\end{array}\right)\left\langle\prod_{j=1}^{r}\left(\frac{(1+t)^{\lambda_{j}}}{1+(1+t)^{\lambda_{j}}}\right)^{\mu_{j}} \mid x^{n-m}\right\rangle \\
& =\left(\begin{array}{c}
n \\
m
\end{array}\right) \hat{S}_{n-m} .
\end{aligned}
$$

Hence, we have the following identities.

Theorem 9 For all $n \geq 0$,

$$
S_{n}(x)=\sum_{m=0}^{n} S_{n-m}\left(\begin{array}{l}
n \\
m
\end{array}\right)(x)_{m} \quad \text { and } \quad \hat{S}_{n}(x)=\sum_{m=0}^{n} \hat{S}_{n-m}\left(\begin{array}{l}
n \\
m
\end{array}\right)(x)_{m} .
$$


Kim et al. Journal of Inequalities and Applications 2014, 2014:324

Page 14 of 16

http://www.journalofinequalitiesandapplications.com/content/2014/1/324

Now, let $S_{n}(x)=\sum_{m=0}^{n} c_{n, m} H_{m}^{(s)}(x \mid \alpha)$ and $\hat{S}_{n}(x)=\sum_{m=0}^{n} \hat{c}_{n, m} H_{m}^{(s)}(x \mid \alpha)$, where $H_{n}^{(s)}(x \mid \alpha) \sim$ $\left(\left(\frac{e^{t}-\alpha}{1-\alpha}\right)^{s}, t\right)$, with $\alpha \neq 1$. Then, by (1.9), (1.10) and (1.11), we obtain

$$
\begin{aligned}
c_{n, m} & =\frac{1}{m !}\left\langle\left(\frac{e^{\log (1+t)}-\alpha}{1-\alpha}\right)^{s} \prod_{j=1}^{r}\left(1+(1+t)^{\lambda_{j}}\right)^{-\mu_{j}}(\log (1+t))^{m} \mid x^{n}\right\rangle \\
& =\frac{1}{m !(1-\alpha)^{s}}\left\langle\prod_{j=1}^{r}\left(1+(1+t)^{\lambda_{j}}\right)^{-\mu_{j}}(\log (1+t))^{m}(1-\alpha+t)^{s} \mid x^{n}\right\rangle \\
& =\frac{1}{m !(1-\alpha)^{s}}\left\langle\prod_{j=1}^{r}\left(1+(1+t)^{\lambda_{j}}\right)^{-\mu_{j}}(\log (1+t))^{m} \mid \sum_{j=0}^{\min \{s, n\}}\left(\begin{array}{l}
s \\
j
\end{array}\right)(1-\alpha) t^{j} x^{n}\right\rangle \\
& =\frac{1}{m !(1-\alpha)^{s}} \sum_{j=0}^{n-m}\left(\begin{array}{l}
s \\
j
\end{array}\right)(1-\alpha)^{s-j}(n)_{j}\left\langle\prod_{j=1}^{r}\left(1+(1+t)^{\lambda_{j}}\right)^{-\mu_{j}}(\log (1+t))^{m} \mid x^{n-j}\right\rangle
\end{aligned}
$$

and by Theorem 8 , we have

$$
\begin{aligned}
c_{n, m} & =\frac{1}{m !(1-\alpha)^{s}} \sum_{j=0}^{n-m}\left(\begin{array}{l}
s \\
j
\end{array}\right)(1-\alpha)^{s-j}(n)_{j}\left(m ! \sum_{\ell=0}^{n-j-m}\left(\begin{array}{c}
n-j \\
\ell
\end{array}\right) S_{1}(n-j-\ell, m) S_{\ell}\right) \\
& =\sum_{j=0}^{n-m} \sum_{\ell=0}^{n-m-j}\left(\begin{array}{c}
s \\
j
\end{array}\right)\left(\begin{array}{c}
n-j \\
\ell
\end{array}\right)(1-\alpha)^{-j}(n)_{j} S_{1}(n-j-\ell, m) S_{\ell} .
\end{aligned}
$$

By (1.9), (1.10) and (1.12), we obtain

$$
\begin{aligned}
\hat{c}_{n, m} & =\frac{1}{m !}\left\langle\left(\frac{e^{\log (1+t)}-\alpha}{1-\alpha}\right) \prod_{j=1}^{s}\left(\frac{(1+t)^{\lambda_{j}}}{1+(1+t)^{\lambda_{j}}}\right)^{\mu_{j}}(\log (1+t))^{m} \mid x^{n}\right\rangle \\
& =\frac{1}{m !(1-\alpha)^{s}}\left\langle\prod_{j=1}^{r}\left(\frac{(1+t)^{\lambda_{j}}}{1+(1+t)^{\lambda_{j}}}\right)^{\mu_{j}}(\log (1+t))^{m} \mid(1-\alpha+t)^{s} x^{n}\right\rangle \\
& =\frac{1}{m !(1-\alpha)^{s}} \sum_{j=0}^{n-m}\left(\begin{array}{l}
s \\
j
\end{array}\right)(1-\alpha)^{s-j}(n)_{j}\left\langle\prod_{j=1}^{r}\left(\frac{(1+t)^{\lambda_{j}}}{1+(1+t)^{\lambda_{j}}}\right)^{\mu_{j}}(\log (1+t))^{m} \mid x^{n-j}\right\rangle
\end{aligned}
$$

and by Theorem 8 , we have

$$
\begin{aligned}
\hat{c}_{n, m} & =\frac{1}{m !(1-\alpha)^{s}} \sum_{j=0}^{n-m}\left(\begin{array}{l}
s \\
j
\end{array}\right)(1-\alpha)^{s-j}(n)_{j}\left(m ! \sum_{\ell=0}^{n-j-m}\left(\begin{array}{c}
n-j \\
\ell
\end{array}\right) S_{1}(n-j-\ell, m) \hat{S}_{\ell}\right) \\
& =\sum_{j=0}^{n-m} \sum_{\ell=0}^{n-m-j}\left(\begin{array}{c}
s \\
j
\end{array}\right)\left(\begin{array}{c}
n-j \\
\ell
\end{array}\right)(1-\alpha)^{-j}(n)_{j} S_{1}(n-j-\ell, m) \hat{S}_{\ell} .
\end{aligned}
$$

Therefore, we can state the following result.

Theorem 10 For all $n \geq 0$,

$$
S_{n}(x)=\sum_{m=0}^{n}\left(\sum_{j=0}^{n-m} \sum_{\ell=0}^{n-m-j}\left(\begin{array}{c}
s \\
j
\end{array}\right)\left(\begin{array}{c}
n-j \\
\ell
\end{array}\right)(1-\alpha)^{-j}(n)_{j} S_{1}(n-j-\ell, m) S_{\ell}\right) H_{m}^{(s)}(x \mid \alpha),
$$




$$
\hat{S}_{n}(x)=\sum_{m=0}^{n}\left(\sum_{j=0}^{n-m} \sum_{\ell=0}^{n-m-j}\left(\begin{array}{l}
s \\
j
\end{array}\right)\left(\begin{array}{c}
n-j \\
\ell
\end{array}\right)(1-\alpha)^{-j}(n)_{j} S_{1}(n-j-\ell, m) \hat{S}_{\ell}\right) H_{m}^{(s)}(x \mid \alpha) .
$$

Finally, we express our polynomials $S_{n}(x)$ and $\hat{S}_{n}(x)$ in terms of Bernoulli polynomials of order $s$. Let $S_{n}(x)=\sum_{m=0}^{n} c_{n, m} B_{m}^{(s)}(x)$ and $\hat{S}_{n}(x)=\sum_{m=0}^{n} \hat{c}_{n, m} B_{m}^{(s)}(x)$, where $B_{n}^{(s)}(x) \sim$ $\left(\left(\frac{e^{t}-1}{t}\right)^{s}, t\right)$. Then, by (1.9), (1.10) and (1.11), we obtain

$$
\begin{aligned}
c_{n, m} & =\frac{1}{m !}\left\langle\left(\frac{e^{\log (1+t)}-1}{\log (1+t)}\right)^{s} \prod_{j=1}^{r}\left(1+(1+t)^{\lambda_{j}}\right)^{-\mu_{j}}(\log (1+t))^{m} \mid x^{n}\right\rangle \\
& =\frac{1}{m !}\left\langle\prod_{j=1}^{r}\left(1+(1+t)^{\lambda_{j}}\right)^{-\mu_{j}}(\log (1+t))^{m} \mid\left(\frac{t}{\log (1+t)}\right)^{s} x^{n}\right\rangle,
\end{aligned}
$$

and by the fact that $\left(\frac{t}{\log (1+t)}\right)^{s}=\sum_{n \geq 0} C_{n}^{(s)} \frac{t^{n}}{n !}$, where $C_{n}^{(s)}$ is the Cauchy number of the first kind of order $s$, we derive

$$
c_{n, m}=\frac{1}{m !} \sum_{i=0}^{n-m}\left(\begin{array}{c}
n \\
i
\end{array}\right) C_{i}^{(s)}\left\langle\prod_{j=1}^{r}\left(1+(1+t)^{\lambda_{j}}\right)^{-\mu_{j}}(\log (1+t))^{m} \mid x^{n-i}\right\rangle,
$$

and by Theorem 8 , we obtain

$$
\begin{aligned}
& c_{n, m}=\frac{1}{m !} \sum_{i=0}^{n-m}\left(\begin{array}{l}
n \\
i
\end{array}\right) C_{i}^{(s)}\left(m ! \sum_{\ell=0}^{n-i-m}\left(\begin{array}{c}
n-i \\
\ell
\end{array}\right) S_{1}(n-i-\ell, m) S_{\ell}\right) \\
& =\sum_{i=0}^{n-m} \sum_{\ell=0}^{n-i-m}\left(\begin{array}{c}
n \\
i
\end{array}\right)\left(\begin{array}{c}
n-i \\
\ell
\end{array}\right) C_{i}^{(s)} S_{1}(n-i-\ell, m) S_{\ell} .
\end{aligned}
$$

Also, by (1.9), (1.10) and (1.12), we obtain

$$
\begin{aligned}
\hat{c}_{n, m} & =\frac{1}{m !}\left\langle\left(\frac{e^{\log (1+t)}-1}{\log (1+t)}\right)^{s} \prod_{j=1}^{r}\left(\frac{(1+t)^{\lambda_{j}}}{1+(1+t)^{\lambda_{j}}}\right)^{\mu_{j}}(\log (1+t))^{m} \mid x^{n}\right\rangle \\
& =\frac{1}{m !}\left\langle\prod_{j=1}^{r}\left(\frac{(1+t)^{\lambda_{j}}}{1+(1+t)^{\lambda_{j}}}\right)^{\mu_{j}}(\log (1+t))^{m} \mid\left(\frac{t}{\log (1+t)}\right)^{s} x^{n}\right\rangle \\
& =\frac{1}{m !} \sum_{i=0}^{n-m}\left(\begin{array}{c}
n \\
i
\end{array}\right) C_{i}^{(s)}\left\langle\prod_{j=1}^{r}\left(\frac{(1+t)^{\lambda_{j}}}{1+(1+t)^{\lambda_{j}}}\right)^{\mu_{j}}(\log (1+t))^{m} \mid x^{n-i}\right\rangle,
\end{aligned}
$$

and by Theorem 8 , we obtain

$$
\begin{aligned}
& c_{n, m}=\frac{1}{m !} \sum_{i=0}^{n-m}\left(\begin{array}{c}
n \\
i
\end{array}\right) C_{i}^{(s)}\left(m ! \sum_{\ell=0}^{n-i-m}\left(\begin{array}{c}
n-i \\
\ell
\end{array}\right) S_{1}(n-i-\ell, m) \hat{S}_{\ell}\right) \\
& =\sum_{i=0}^{n-m} \sum_{\ell=0}^{n-i-m}\left(\begin{array}{c}
n \\
i
\end{array}\right)\left(\begin{array}{c}
n-i \\
\ell
\end{array}\right) C_{i}^{(s)} S_{1}(n-i-\ell, m) \hat{S}_{\ell} .
\end{aligned}
$$

Hence, we have the following identities. 
Theorem 11 For all $n \geq 0$,

$$
\begin{aligned}
& S_{n}(x)=\sum_{m=0}^{n}\left(\sum_{j=0}^{n-m} \sum_{\ell=0}^{n-m-j}\left(\begin{array}{l}
n \\
j
\end{array}\right)\left(\begin{array}{c}
n-j \\
\ell
\end{array}\right) C_{j}^{(s)} S_{1}(n-j-\ell, m) S_{\ell}\right) B_{m}^{(s)}(x), \\
& \hat{S}_{n}(x)=\sum_{m=0}^{n}\left(\sum_{j=0}^{n-m} \sum_{\ell=0}^{n-m-j}\left(\begin{array}{c}
n \\
j
\end{array}\right)\left(\begin{array}{c}
n-j \\
\ell
\end{array}\right) C_{j}^{(s)} S_{1}(n-j-\ell, m) \hat{S}_{\ell}\right) B_{m}^{(s)}(x) .
\end{aligned}
$$

\section{Competing interests}

The authors declare that they have no competing interests.

\section{Authors' contributions}

All authors contributed equally to this work. All authors read and approved the final manuscript.

\section{Author details}

'Department of Mathematics, Sogang University, Seoul, 121-742, S. Korea. ${ }^{2}$ Department of Mathematics, Kwangwoon University, Seoul, 139-701, S. Korea. ${ }^{3}$ Department of Mathematics, University of Haifa, Haifa, 3498838, Israel. ${ }^{4}$ Department of Applied Mathematics, Pukyong National University, Pusan, S. Korea.

\section{Acknowledgements}

The authors would like to thank the referees for their valuable comments. This work was supported by the National Research Foundation of Korea (NRF) grant funded by the Korea government (MOE) (No. 2012R1A1A2003786) and was partially supported by Kwangwoon University in 2014

Received: 23 June 2014 Accepted: 1 August 2014 Published: 22 Aug 2014

\section{References}

1. Agapito Ruiz, J: Riordan arrays from an umbral symbolic viewpoint. Bol. Soc. Port. Mat., Special Issue, 5-8 (2012)

2. Di Bucchianico, A, Loeb, D: A selected survey of umbral calculus. Electron. J. Comb. 2, DS3 (2000)

3. Dattoli, G, Levi, D, Winternitz, P: Heisenberg algebra, umbral calculus and orthogonal polynomials. J. Math. Phys. 49(5), 053509 (2008)

4. Dere, R, Simsek, Y: Applications of umbral algebra to some special polynomials. Adv. Stud. Contemp. Math. 22(3), 433-438 (2012)

5. Kim, DS, Kim, T, Lee, S-H, Rim, S-H: Frobenius-Euler polynomials and umbral calculus in the $p$-adic case. Adv. Differ Equ. 2012, Article ID 222 (2012)

6. Kim, T: Identities involving Laguerre polynomials derived from umbral calculus. Russ. J. Math. Phys. 21(1), 36-45 (2014)

7. Kim, T, Kim, DS, Mansour, T, Rim, S-H, Schork, M: Umbral calculus and Sheffer sequences of polynomials. J. Math. Phys. 54(8), $083504(2013)$

8. Kwaśniewski, AK: q-Quantum plane, $\psi(q)$-umbral calculus, and all that. Quantum groups and integrable systems (Prague, 2001). Czechoslov. J. Phys. 51(12), 1368-1373 (2001)

9. Levi, D, Tempesta, P, Winternitz, P: Umbral calculus, difference equations and the discrete Schrödinger equation. J. Math. Phys. 45(11), 4077-4105 (2004)

10. Wilson, BG, Rogers, FG: Umbral calculus and the theory of multispecies nonideal gases. Physica A 139, 359-386 (1986)

11. Biedenharn, LC, Gustafson, RA, Lohe, MA, Louck, JD, Milne, SC: Special functions and group theory in theoretical physics. In: Special Functions: Group Theoretical Aspects and Applications. Math. Appl., pp. 129-162. Reidel, Dordrecht (1984)

12. Biedenharn, LC, Gustafson, RA, Milne, SC: An umbral calculus for polynomials characterizing U(n) tensor products. Adv. Math. 51, 36-90 (1984)

13. Blasiak, P: Combinatorics of Boson normal ordering and some applications. Concepts Phys. 1, 177-278 (2004)

14. Blasiak, P, Dattoli, G, Horzela, A, Penson, KA: Representations of monomiality principle with Sheffer-type polynomials and boson normal ordering. Phys. Lett. A 352, 7-12 (2006)

15. Gzyl, H: Hamilton Flows and Evolution Semigroups. Pitman Research Notes in Mathematics Series, vol. 239 (1990)

16. Morikawa, H: On differential polynomials I. Nagoya Math. J. 148, 39-72 (1997)

17. Morikawa, H: On differential polynomials II. Nagoya Math. J. 148, 73-112 (1997)

18. Zachos, CK: Umbral deformations on discrete space-time. Int. J. Mod. Phys. A 23(13), 2005-2014 (2008)

19. Roman, S: The Umbral Calculus. Dover, New York (2005)

20. Kim, DS, Kim, T: Applications of umbral calculus associated with $p$-adic invariant integrals on $\mathbb{Z}_{p}$. Abstr. Appl. Anal. 2012, Article ID 865721 (2012)

21. Kim, DS, Kim, T: Some identities of Frobenius-Euler polynomials arising from umbral calculus. Adv. Differ. Equ. 2012, Article ID 196 (2012)

22. Roman, S: More on the umbral calculus, with emphasis on the q-umbral calculus. J. Math. Anal. Appl. 107, 222-254 (1985)

10.1186/1029-242X-2014-324

Cite this article as: Kim et al.: Barnes-type Peters polynomial with umbral calculus viewpoint. Journal of Inequalities and Applications 2014, 2014:324 